\title{
On the Plasmonic Properties of Double-Deck Graphene Nanoribbon Structure in Mid-Infrared Regime
}

\section{Yue Zhang}

Dalian University

\section{Dandan Dong}

Dalian University

\section{Tao Xiong}

Dalian University

\section{Wei Wang}

Dalian University

Cheng Sun ( $\nabla$ suncheng@dlu.edu.cn )

Dalian Univeristy https://orcid.org/0000-0002-7014-7126

\section{Research Article}

Keywords: Plasmonics, Graphene nanoribbon, Mid infrared, FDTD

Posted Date: May 10th, 2021

DOl: https://doi.org/10.21203/rs.3.rs-474264/v1

License: (c) (1) This work is licensed under a Creative Commons Attribution 4.0 International License. Read Full License

Version of Record: A version of this preprint was published at Plasmonics on July 28th, 2021. See the published version at https://doi.org/10.1007/s11468-021-01505-7. 


\title{
On the plasmonic properties of double-deck graphene nanoribbon structure in mid-infrared regime
}

\author{
Yue Zhang, Dandan Dong, Tao Xiong, Wei Wang, Cheng Sun* \\ College of Physical Science and Technology, Dalian University, 116622, China
}

\begin{abstract}
In this work a double-deck structure consisting of graphene nanoribbons is proposed, and the plasmonic properties of the system is investigated at midinfrared wavelengths. In the structure, two graphene nanoribbon arrays with different parameters are coated on both sides of a glass substrate. With respectively varying the Fermi energies, the layer numbers of the graphenes, and the widths of the ribbons, the light transmittance is studied in the wavelength region from 5 to $30 \mu \mathrm{m}$, via the finite difference time domain method. Multiple plasmonic resonances are revealed in the double-deck graphenes. It is shown that the plasmonic resonance properties including the resonance wavelengths, the peak intensities, and the peak widths can be adjusted by changing the parameters of the graphenes. The results suggest that the double-deck graphene nanoribbon structure proposed in this work be implemented in designs of plasmonic devices in mid-infrared regime.
\end{abstract}

Key words: Plasmonics; Graphene nanoribbon; Mid infrared; FDTD

${ }^{*}$ Corresponding author.

Email address: suncheng@dlu.edu.cn(Cheng Sun). 


\section{Introduction}

Existing plasmonic devices that are based on conventional materials usually have a relatively low performance of either slow response time or limited tunability[1-3]. Graphene, the single layer of graphite, consists of a hexagonally arranged carbon atoms. Thanks to its $\mathrm{SP}^{2}$ bonds, graphene possesses many unique electronic properties, and hence graphene-based nanostructures provide us with a great platform to design plasmonic devices with great properties such as high speed and broad tunablility, etc.[4] To date, several graphene-based structures have been proposed and corresponding plasmonic devices have been investigated. For instance, tunable infrared plasmonic devices by using graphene-insulator stacks were experimentally demonstrated[5]. Graphene ring based structures for highly tunable optical antennas in teraherz range were reported[6]. In addition, a switched-beam graphene nanoantennas over glass substrate was also proposed, and the reconfigurability of the nanoantenna was achieved by tuning the graphene conductivity through its chemical potential[7]. Besides, the plasmonic properties of a graphene core-shell nanostructure was numerically simulated, and the plasmonic resonance effect associated with the graphene shell was presented[8]. A design of graphene-based plasmonic waveguide was reported, and its transmission properties were studied; a forbidden bandgap at $\mathrm{THz}$ frequencies was shown, which was similar to a conventional photonic crystal[9]. Recently, a graphene-based plasmonic metamaterial absorber was presented, which operated as an ultra-compact optical gas sensor in the infrared region, and three distinct plasmonic resonances were addressed using the finite element $\operatorname{method}[10]$.

Nowadays, graphene-related plasmonic structures and devices that operate in the mid-infrared regime have drawn great attention, since this wavelength range is important in many promising applications, including sensing, imaging, remote detecting, THz antennas, as well as optical spectroscopy[1113]. Due to the periodic oscillations of electrons in graphene, highly confined plasmonic waves may exist and they propagate in the graphene plane[14]. It is known that, in the mid-infrared regime, the plasmonic wavevector is much larger than that of the incident light, causing a highly confined electric field[15]. To overcome this great mismatch under the irradiation of the midinfrared incident light, array structures consisting of graphene nanoribbons were proposed. Thus, instead of propagating in a continuous graphene plane, the plasmonic modes are confined in the ribbons[16]. A plasmonic resonance 
may occur when the period of the plasmonic wave matches that of the ribbons. So far, various graphene ribbon based structures have been designed, in order to work at mid-infrared wavelengths. For example, composite arrays of graphene ribbons were investigated, and the tunability of plasmonic resonance in the mid-infrared wavelength range was domenstrated[17]. Moreover, a graphene ribbon and double layer sheets structure was theoretically studied, and a tunable strong coupling mechanism was also demonstrated[18]. Besides, Terahertz plasmonic logic gates were achieved using graphene nanoribbon waveguides[19]. Recently, the circular dichroism (CD) effects in the mid-infrared region of a metamaterial consisting of graphene ribbons and metal array was probed; it was shown that the intensity and peak position of the CD response could be controlled by the Fermi energy of graphene[20]. In this work, we propose a graphene nanoribbon based double-deck structure, and demonstrate the tunability of plasmonic resonances revealed in the light transmittance curve in the mid-infrared wavelength region from 5 to $30 \mu \mathrm{m}$.

\section{Structure and method}

As shown in Fig.1, a double-deck structure comprised of graphene nanoribbons was proposed. In the structure, two sets of graphene ribbon arrays (indicated in Top and Bottom, respectively) were placed on both sides of a glass substrate. As indicated in the figure, the array period along the $x$-axis was $\Delta$, and the ribbon widths were $d_{T}$ and $d_{B}$. The thickness of the glass spacer was $h$, and it was assumed to be $200 \mathrm{~nm}$. In this work, the Fermi energies were labeled $\mu_{c}^{T}$ and $\mu_{c}^{B}$, and the layer numbers were $N_{T}$ and $N_{B}$ for Top and Bottom graphenes, respectively.

In this work the finite difference time domain (FDTD) method was used[21]. In all calculations, a plane-wave light was normally incident in a wavelength range of $5-30 \mu \mathrm{m}$ (along the - z-axis shown in Fig.1), and the electric vector of the light was kept $x$-polarized. In the $z$ direction, the perfect match layer (PML) boundary condition was used. In the $x-y$ plane where the graphene ribbon arrays were place, periodic boundary conditions were utilized. In order to avoid the artifacts that might be induced by the simulation method, the mesh size was always smaller than $1 / 10$ of the shortest wavelength studied in the simulation region of non plasmon-carrying media. In this work a plane monitor in the $x-y$ plane that measured the light transmittance was placed right beneath Bottom graphene (not shown in Fig.1 for clarity). The 


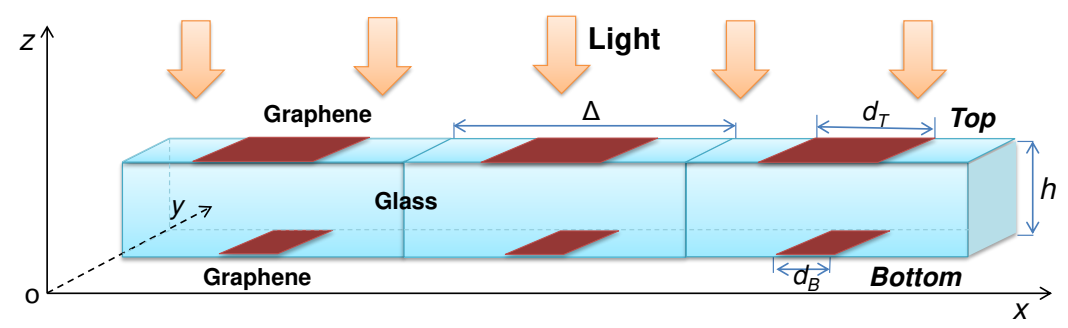

Figure 1: Schematic of the double-deck structure consisting of graphene nanoribbons. A plane-wave light is normally incident along the $-z$-axis. Two sets of graphene ribbon arrays (indicated in Top and Bottom) are placed on both sides of a glass substrate. The thickness of the glass is $h$. Note that along the $x$-axis, the basic structure in one period repeats infinitely to fulfil the array condition; only three periods are drawn in the figure for brevity. The length of one period along the $x$-axis is $\Delta$. The ribbons are infinite in the $y$ direction. The widths of the ribbons are $d_{T}$ and $d_{B}$, respectively.

electric field distributions of the double-deck structure were also collected via plane monitors that were positioned at the surfaces of both graphenes.

For a single-layer graphene, the optical constant may be determined from its surface conductivity, $\sigma$, by the following relationship,

$$
\sigma=\sigma_{\text {intra }}+\sigma_{\text {inter }}
$$

where $\sigma_{\text {intra }}$ and $\sigma_{\text {inter }}$ are the intraband and interband terms, respectively. The intraband term may be given by the equation below[22]

$$
\sigma_{\text {intra }}=-i \frac{e^{2} k_{B} T}{\pi \hbar^{2}(\omega-i 2 \Gamma)}\left[\frac{\mu_{c}}{k_{B} T}+2 \ln \left(e^{-\mu_{c} / k_{B} T}+1\right)\right]
$$

In Eq.2, $e$ is the electron charge, $k_{B}$ is the Boltzmann's constant, $\hbar$ is the reduced Planck's constant, and $T$ is the temperature and it was set $300 \mathrm{~K}$ in this work. $\omega$ is the angular frequency of the incident light, and $\mu_{c}$ is the Fermi energy of graphene. $\Gamma$ is the scattering rate, which is normally determined by experiments. Based on typical values of the carrier mobility, the phenomenological scattering rate was assumed to be $\Gamma=0.11 \mathrm{meV}$ in this work $[23,24]$; a similar value of $\Gamma$ was also used in literature [25].

Referring to Eq.1, the interband term, $\sigma_{\text {inter }}$, has no analytical solutions, and yet an approximation can be made when $k_{B} T \ll \mu_{c}$, $\hbar \omega[26]$. In this work, 
the above condition was held, and the interband term is thus approximately expressed as

$$
\sigma_{\text {inter }}=-i \frac{e^{2}}{4 \pi \hbar} \ln \left[\frac{2 \mu_{c}-(\omega-i 2 \Gamma) \hbar}{2 \mu_{c}+(\omega-i 2 \Gamma) \hbar}\right]
$$

Based on the surface conductivity for a single-layer graphene, the conductivity for a multi-layer graphene may be determined by considering the layer number $(N)$, and hence the value of $N \times \sigma$ can be used[27].

\section{Results and discussions}

In this work, the plasmonic properties of the graphene double-deck system are mainly characterized by the light transmittance. As an example, Fig.2 demonstrates the simulated result of the transmittance under the irradiation of the plane-wave light in the wavelength range of $5-30 \mu \mathrm{m}$, in which the graphene ribbon parameters were $\mu_{c}^{T}=0.20 \mathrm{eV}, \mu_{c}^{B}=0.55 \mathrm{eV}, N_{T}=N_{B}=$ $1, d_{T}=300 \mathrm{~nm}, d_{B}=100 \mathrm{~nm}$, and $\Delta=600 \mathrm{~nm}$.

Three peaks (labeled $B, T_{\alpha}$ and $T_{\beta}$, respectively) are revealed in the wavelength dependent transmittance curve shown in Fig.2. As known to the community, the appearance of the peaks is the manifestation of plasmonic resonance effects occurring in graphene nanoribbons under the light irradiation; this phenomenon was also reported and discussed in literature[15, 25]. In detail, plasmonic resonance may take place once the period of the light-induced plasmonic wave in graphene matches the dimension of the graphene array. On resonance, the light's energy is substantially dissipated while the excited plasmonic wave propagates in the graphene, manifesting itself into peaks in the transmittance curve. In previous work, it has been shown that the characteristics of the plasmonic wave depends on the properties of graphene nanoribbons, therefore it is worthwhile to probe the double-deck system, by varying the graphene parameters including the Fermi energy, the layer number, and the ribbon width.

Referring to Fig.2 again, the observation of multiple peaks may be accounted for by the resonance effect associated with different plasmonic modes that propagate in graphene nanostructures. Similar effects were also reported previously in Ref.[28] where the plasmon induced transparency in two coupled graphene gratings were studied in the mid-infrared region, and a small peak was revealed in the transmittance curve; it was also found in the literature that as with the large resonance wavelength, the small peak induced 


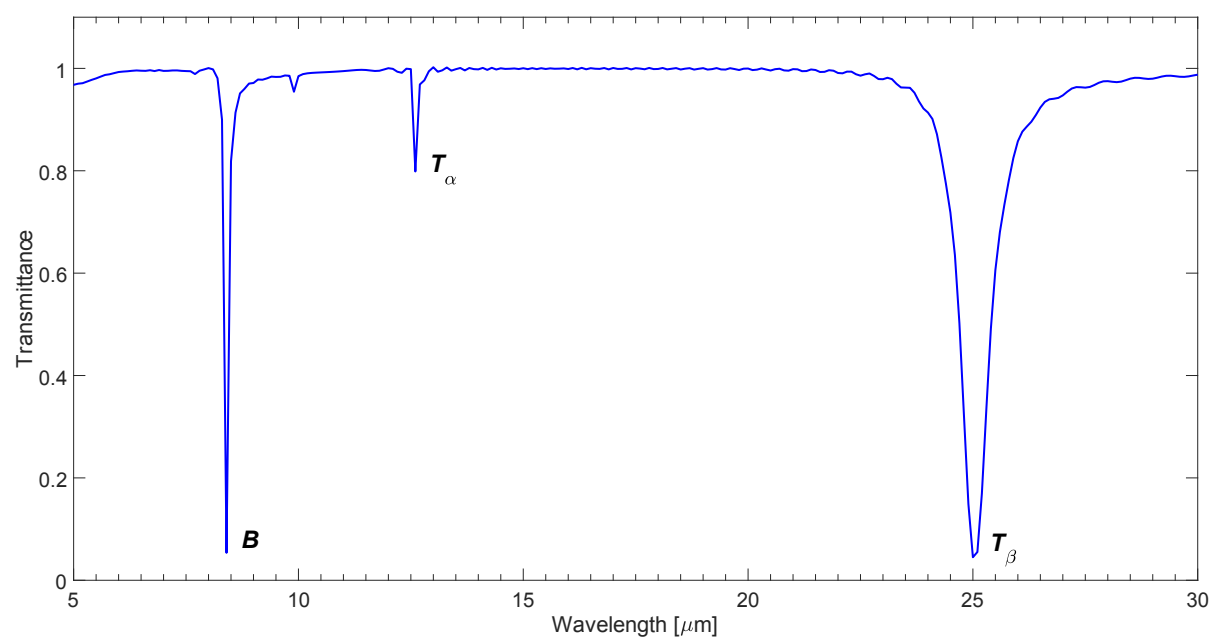

Figure 2: Wavelength-dependent light transmittance determined from the structure shown in Fig.1. In the simulation $\mu_{c}^{T}=0.20 \mathrm{eV}, \mu_{c}^{B}=0.55 \mathrm{eV}, N_{T}=N_{B}=1, d_{T}=300 \mathrm{~nm}, d_{B}$ $=100 \mathrm{~nm}$, and $\Delta=600 \mathrm{~nm}$.

by the coupling of two modes was also tunable as the optical properties of the graphene was changed, and that the intensity of the small peak could be as great as the large resonance peak in certain conditions. In this work, the ribbon arrays on both sides of the glass were deliberately set different, so that the multiple resonance effect may be clearly revealed in the transmittance curve.

To probe the different plasmonic modes of the system, the distributions of the electric field intensity at the surfaces of both graphene ribbons were computed, and the results at resonance wavelengths are shown in Fig.3. Note that in Fig.3a, for Bottom graphene the electric field is given at the wavelength of peak $B$ only, while the fields at peaks $T_{\alpha}$ and $T_{\beta}$ are not shown since their intensities are negligible. This infers the fact that resonance peak $B$ arises from the plamonic mode associated with Bottom graphene. Similarly, in Figs.3b and 3c, the Top graphene's field is only shown at peaks $T_{\alpha}$ and $T_{\beta}$, whereas its field at peak $B$ is also weak and is not given here. This observation indicates that resonance peaks $T_{\alpha}$ and $T_{\beta}$ are related to Top graphene. Besides, the difference in the distributions between Fig.3b and Fig.3c implies that there are two different plasmonic modes in Top graphene, which give rise to two resonance peaks of $T_{\alpha}$ and $T_{\beta}$, respectively.

First, the dependence of the plasmonic resonances on the Fermi energy of 

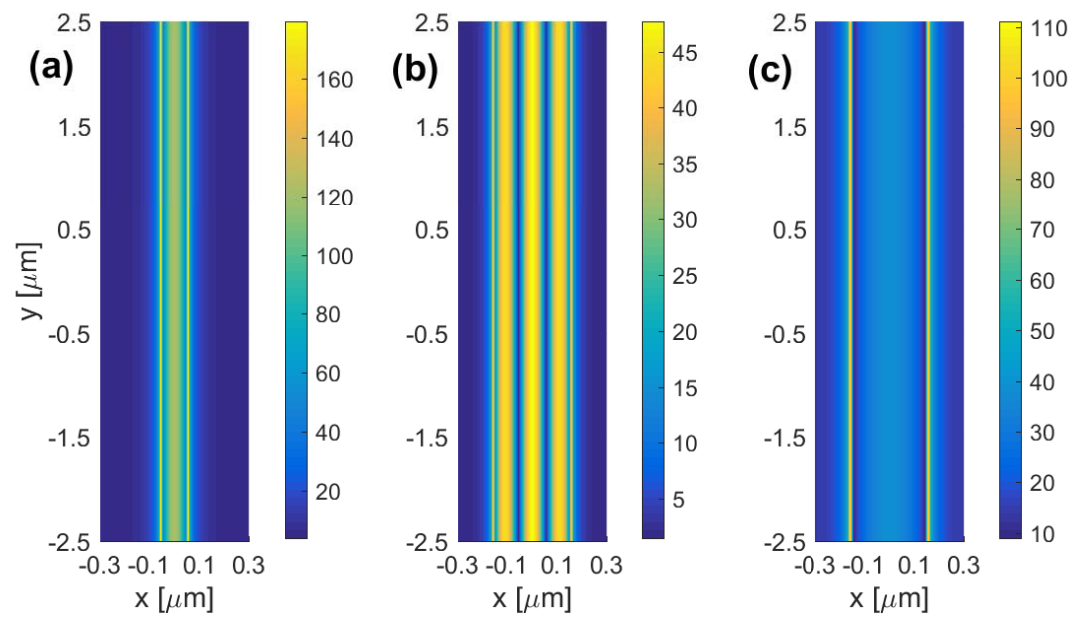

Figure 3: Distribution of the electric field intensity at the surface of (a) Bottom graphene, and (b) and (c) Top graphene in the $x-y$ plane. The wavelength of the incident light was at (a) peak $B$, (b) peak $T_{\alpha}$, and (c) peak $T_{\beta}$ indicated in Fig.2. In the simulation $\mu_{c}^{T}=$ $0.20 \mathrm{eV}, \mu_{c}^{B}=0.55 \mathrm{eV}, N_{T}=N_{B}=1, d_{T}=300 \mathrm{~nm}, d_{B}=100 \mathrm{~nm}$, and $\Delta=600 \mathrm{~nm}$. Along the $x$-axis, the range from -0.3 to $0.3 \mu \mathrm{m}$ represents one period of the array, where the Bottom graphene ribbon was located within $(-0.050 .05) \mu \mathrm{m}$ and the Top ribbon within $(-0.150 .15) \mu \mathrm{m}$. Note that the intensity of the color bar is different in each figure. 


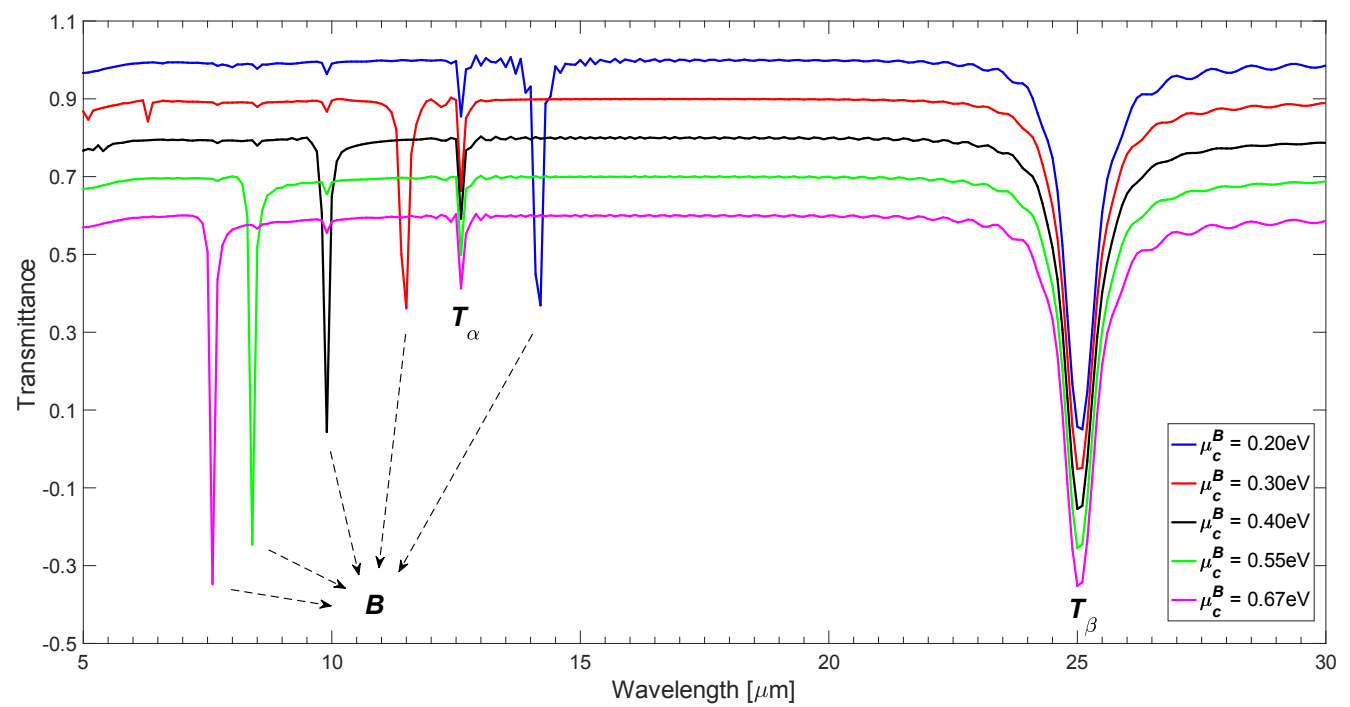

Figure 4: Wavelength-dependent light transmittance determined from the structure shown in Fig.1. In the simulation $\mu_{c}^{T}=0.20 \mathrm{eV}, N_{T}=N_{B}=1, d_{T}=300 \mathrm{~nm}, d_{B}=100 \mathrm{~nm}$, and $\Delta$ $=600 \mathrm{~nm}$. For clarity, the curves are presented with an offset in the $y$-axis: $\mu_{c}^{B}=0.30 \mathrm{eV}$ is offset by -0.1 , the $0.40 \mathrm{eV}$ the -0.2 , the $0.55 \mathrm{eV}$ the -0.3 , and the $0.67 \mathrm{eV}$ the -0.4 .

Bottom graphene was investigated, and the resultant transmittance curves are presented in Fig.4. In the figure, $\mu_{c}^{T}=0.20 \mathrm{eV}$ was kept the same, and $\mu_{c}^{B}$ was varied from 0.20 to $0.67 \mathrm{eV}$. It is obvious from Fig.4 that peak $B$ is blue shifted as the Fermi energy, $\mu_{c}^{B}$, is raised. Note that peaks $T_{\alpha}$ and $T_{\beta}$ are almost unchanged, and this verifies that the aforementioned fact that these two peaks are related to Top graphene only. Focusing on peak $B$, the resonance properties are quantitatively probed via the values shown in Fig.5. In the figure, the resonance wavelength (labeled $\lambda_{B}$ ), the peak intensity (denoted in $I_{B}$ ), as well as the full width at half maximum (FWHM) of the peak (indicated in $\delta_{B}$ ) are summarized in Figs.5a, 5b, and 5c, respectively.

Regarding Fig.5a, with increasing the value of $\mu_{c}^{B}$ the decreasing of $\lambda_{B}$ from 14.2 to $7.6 \mu \mathrm{m}$ is observed, correlating to the blue-shift trend indicated in Fig.4. This may be attributed to the increase in the graphene's surface conductivity, as its Fermi energy is raised; similar effects were also observed and addressed in previous work in Ref.[17]. Referring to Fig.5b, it is obvious that the intensity is reduced from roughly 0.46 to 0.05 as $\mu_{c}^{B}$ is increased. It is shown in Fig.5c that the FWHM is slightly decreased from 0.21 to $0.12 \mu \mathrm{m}$ with increasing the Fermi energy. 

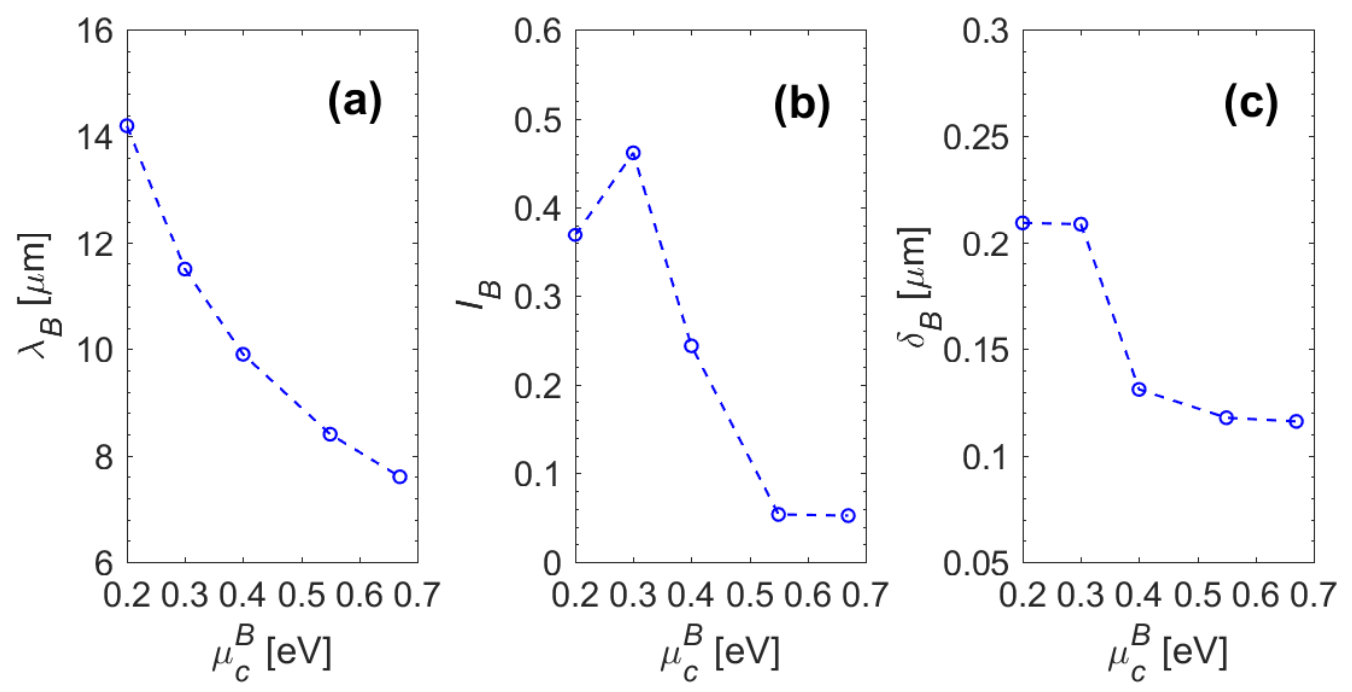

Figure 5: The values of the resonance wavelength $\left(\lambda_{B}\right)$, the peak intensity $\left(I_{B}\right)$, and the FWHM $\left(\delta_{B}\right)$, determined from peak $B$ shown in Fig.4.

As with the study of Bottom graphene's Fermi energy, the effects of the Fermi energy of Top graphene were also examined, and the corresponding transmittance curves are illustrated in Fig.6, in which $\mu_{c}^{B}$ was fixed to be $0.55 \mathrm{eV}$, while $\mu_{c}^{T}$ was changed from 0.20 to $0.50 \mathrm{eV}$. Similar to the trend observed in Fig.4, with increasing the value of $\mu_{c}^{T}$, a blue shift fashion is also witnessed for both peaks $T_{\alpha}$ and $T_{\beta}$. Note that peak $B$ remains the same. Corresponding to peaks $T_{\alpha}$ and $T_{\beta}$ in Fig.6, the values of the resonance wavelengths, the peak intensities and the FWHMs were measured, and the results are plotted in Fig.7. It is clear from Fig.7a that both resonance wavelengths descends (from 12.6 to $7.8 \mu \mathrm{m}$ for $T_{\alpha}$, and from 25.0 to $15.8 \mu \mathrm{m}$ for $T_{\beta}$ ) as $\mu_{c}^{T}$ ascends, correlating to the blue shift behavior addressed above. Revealed in Fig.7b, with increasing the value of $\mu_{c}^{T}$, the intensity of peak $T_{\alpha}$ oscillates between 0.8 and 0.6 , while peak $T_{\beta}$ remains almost at 0 . It is shown in Fig.7c that both FWHMs have subtle variations, respectively, that is, about $0.1 \mu \mathrm{m}$ for $T_{\alpha}$ and around $0.7 \mu \mathrm{m}$ for $T_{\beta}$.

Apart from Fermi energy, layer number is another parameter that may affect the surface conductivity and hence the optical properties of graphene systems. To further probe the plasmonic properties of the double-deck graphene nanoribbons, a set of simulations were also performed as the layer number 


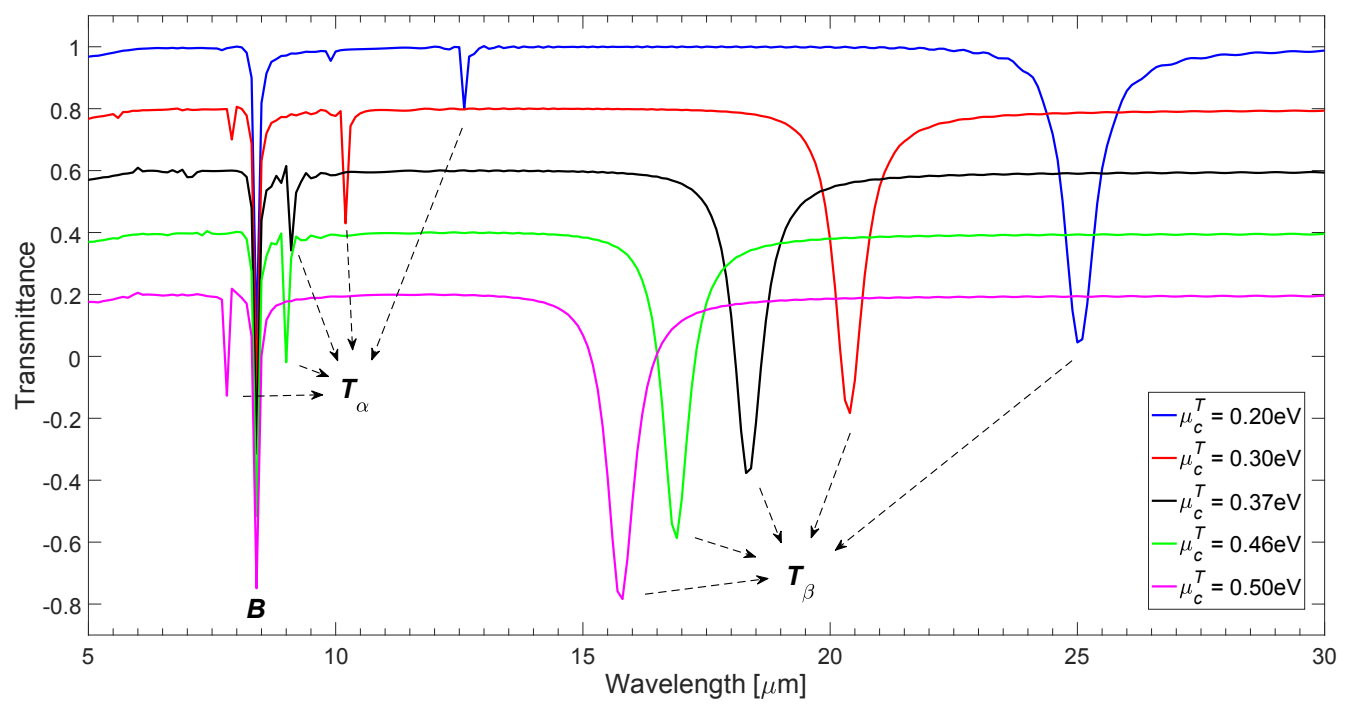

Figure 6: Wavelength-dependent light transmittance determined from the structure shown in Fig.1. In the simulation $\mu_{c}^{B}=0.55 \mathrm{eV}, N_{T}=N_{B}=1, d_{T}=300 \mathrm{~nm}, d_{B}=100 \mathrm{~nm}$, and $\Delta$ $=600 \mathrm{~nm}$. For clarity, the curves are presented with an offset in the $y$-axis: $\mu_{c}^{T}=0.30 \mathrm{eV}$ is offset by -0.2 , the $0.37 \mathrm{eV}$ the -0.4 , the $0.46 \mathrm{eV}$ the -0.6 , and the $0.50 \mathrm{eV}$ the -0.8 .
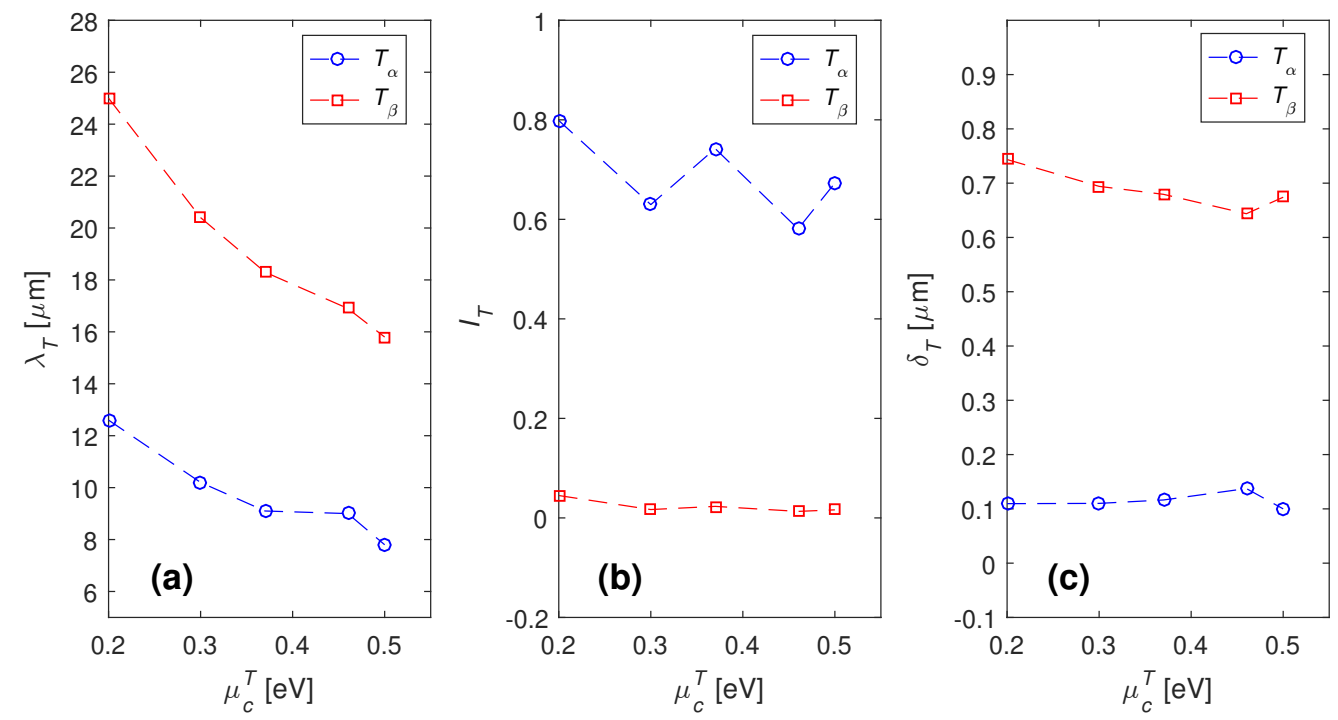

Figure 7: The values of the resonance wavelengths $\left(\lambda_{T}\right)$, the peak intensities $\left(I_{T}\right)$, and the FWHMs $\left(\delta_{T}\right)$, determined from peaks $T_{\alpha}$ and $T_{\beta}$ shown in Fig.6. 


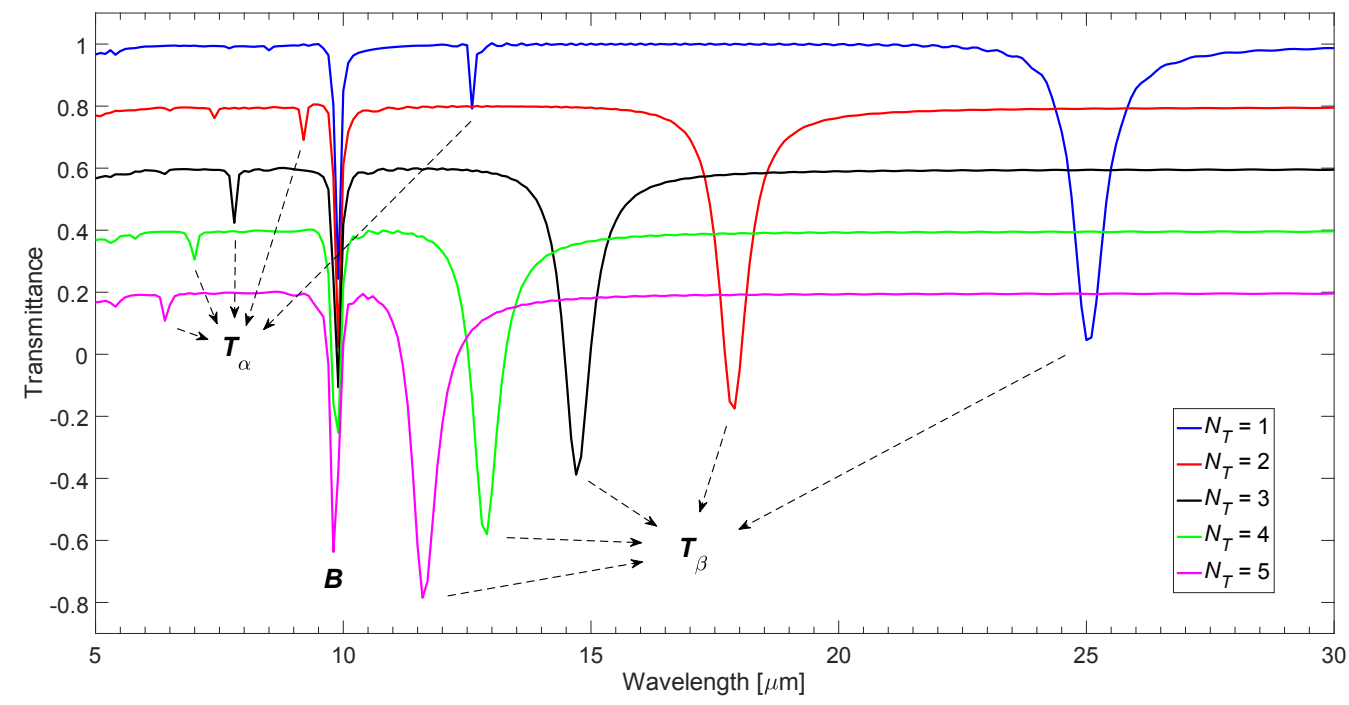

Figure 8: Wavelength-dependent light transmittance determined from the structure shown in Fig.1. In the simulation $\mu_{c}^{T}=0.20 \mathrm{eV}, \mu_{c}^{B}=0.40 \mathrm{eV}, N_{B}=1, d_{T}=300 \mathrm{~nm}, d_{B}=100 \mathrm{~nm}$, and $\Delta=600 \mathrm{~nm}$. For clarity, the curves are presented with an offset in the $y$-axis: $N_{T}=$ 2 is offset by -0.2 , the 3 the -0.4 , the 4 the -0.6 , and the 5 the -0.8 .

of Top graphene was changed from $N_{T}=1$ to 5 . The transmittance curves are presented in Fig. 8 where $N_{B}$ was kept to be $1, \mu_{c}^{T}=0.20 \mathrm{eV}$, and $\mu_{c}^{B}=$ $0.40 \mathrm{eV}$. It is clear from Fig.8 that peaks $T_{\alpha}$ and $T_{\beta}$ are both blue shifted as $N_{T}$ is increased, while peak $B$ remains constant. The observation of the blue shift in peaks $T_{\alpha}$ and $T_{\beta}$ reveals that the energies associated with the plasmonic modes in Top graphene are increased, since the surface conductivity is enhanced as the layer number is increased; similar effect was also witnessed in previous work[17].

From peaks $T_{\alpha}$ and $T_{\beta}$ in Fig.8, the resonance properties were also quantified, and the resultant values are detailed in Fig.9. A clear decline in both resonance wavelengths is shown in Fig.9a. With increasing the layer number of $N_{T}$ from 1 to $5, \lambda_{T}$ is decreased from 12.6 to $6.4 \mu \mathrm{m}$ for $T_{\alpha}$, and from 25.0 to $11.6 \mu \mathrm{m}$ for $T_{\beta}$. Referring to Fig.9b, the intensity of peak $T_{\alpha}$ appears little variations between 0.8 and 0.9 , and $I_{T}$ for peak $T_{\beta}$ keeps almost unchanged around 0. From Fig.9c it is revealed that the FWHM of peak $T_{\alpha}$ gives a subtle increase from 0.10 to $0.15 \mu \mathrm{m}$, while that of peak $T_{\alpha}$ presents a clear reduction from 0.74 to $0.59 \mu \mathrm{m}$.

In addition to the Fermi energy and the layer number, the effect of the 

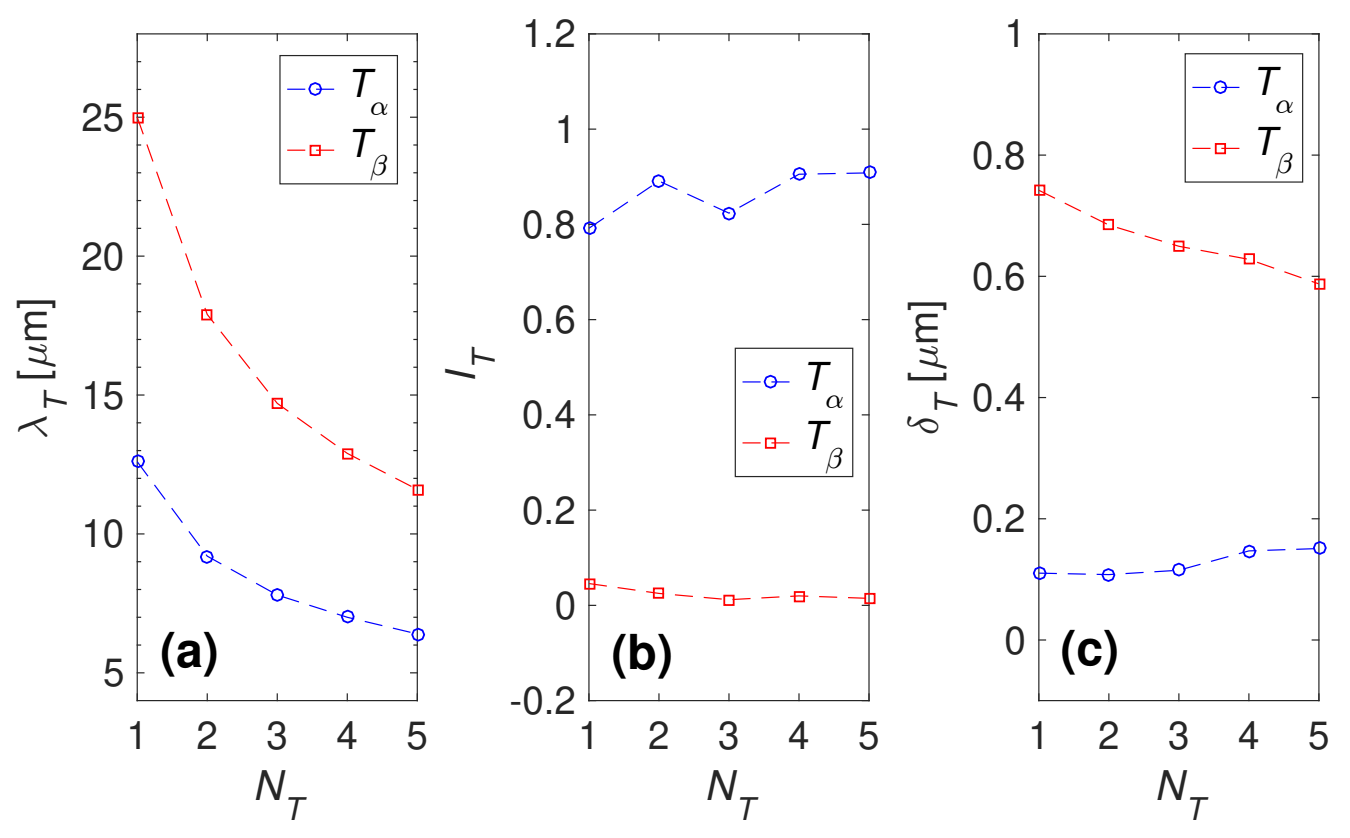

Figure 9: The values of the resonance wavelengths $\left(\lambda_{T}\right)$, the peak intensities $\left(I_{T}\right)$, and the FWHMs $\left(\delta_{T}\right)$, determined from peaks $T_{\alpha}$ and $T_{\beta}$ shown in Fig.8. 
ribbon width of Top graphene on the plasmonic properties of the system was also studied, and the calculated transmittance curves under a variety of $d_{T}$ are shown in Fig.10. In the figure $d_{B}=100 \mathrm{~nm}, N_{T}=N_{B}=1, \mu_{T}=0.20 \mathrm{eV}$, and $\mu_{B}=0.40 \mathrm{eV}$. By examining Fig.10, it is evident that resonance peaks $T_{\alpha}$ and $T_{\beta}$ are both red shifted with broadening the ribbon width of Top graphene, whereas resonance peak $B$ remains unchanged. Based on peaks $T_{\alpha}$ and $T_{\beta}$, the values of $\lambda_{T}, I_{T}$, and $\delta_{T}$ were determined, and are given in Fig.11.

Regarding Fig.11a, the resonance wavelengths of both peaks are increased as the ribbon width is broadened. This is consistent with the fact the period of the plasmonic wave that propagates in graphene is increased as the ribbon width is increased, thus the plasmonic resonance occurs at the wavelength with greater values; this effect was also revealed in other structures shown in literature[17]. In Fig.11a, the detailed increase of $\lambda_{T}$ is measured to be from 9.3 to $13.3 \mu \mathrm{m}$ for $T_{\alpha}$, and from 17.2 to $27.0 \mu \mathrm{m}$ for $T_{\beta}$. Based on Fig.11b, it is observed that the intensities of two peaks are both reduced as the width is increased; it is from 0.92 to 0.76 for $T_{\alpha}$, and from 0.18 to 0.03 for $T_{\beta}$. Referring to Fig.11c, the FWHM of peak $T_{\alpha}$ is nearly unchanged around $0.1 \mu \mathrm{m}$, while that of peak $T_{\beta}$ is substantially increased from 0.26 to $0.95 \mu \mathrm{m}$.

\section{Conclusions}

In this work, a double-deck structure composed of graphene nanoribbons has been proposed, and the plasmonic charateristics have been investigated. In the mid-infrared range, the light wavelength dependent transmittance curves have been numerically simulated via the FDTD method. In the transmittance curves, three peaks have been identified, which are correlated to the plasmonic resonances occurring on both sides of the graphene nanoribbons. The values of the peak properties have been determined, including the resonance wavelength, the peak intensity, as well as the FWHM. Based on the resonance peaks, the effects of the plasmonic properties of the system have been probed with a variety of graphene parameters such as Fermi energies, layer numbers, and ribbon widths. The results indicate that the plasmonic resonance wavelengths can systematically be adjusted in the wavelength regime of 5 to $30 \mu \mathrm{m}$, by varying the graphene parameters studied in this work. It is also suggested that the resonance properties be tuned by respectively changing Top or Bottom graphene in this double-deck nanostructure. 


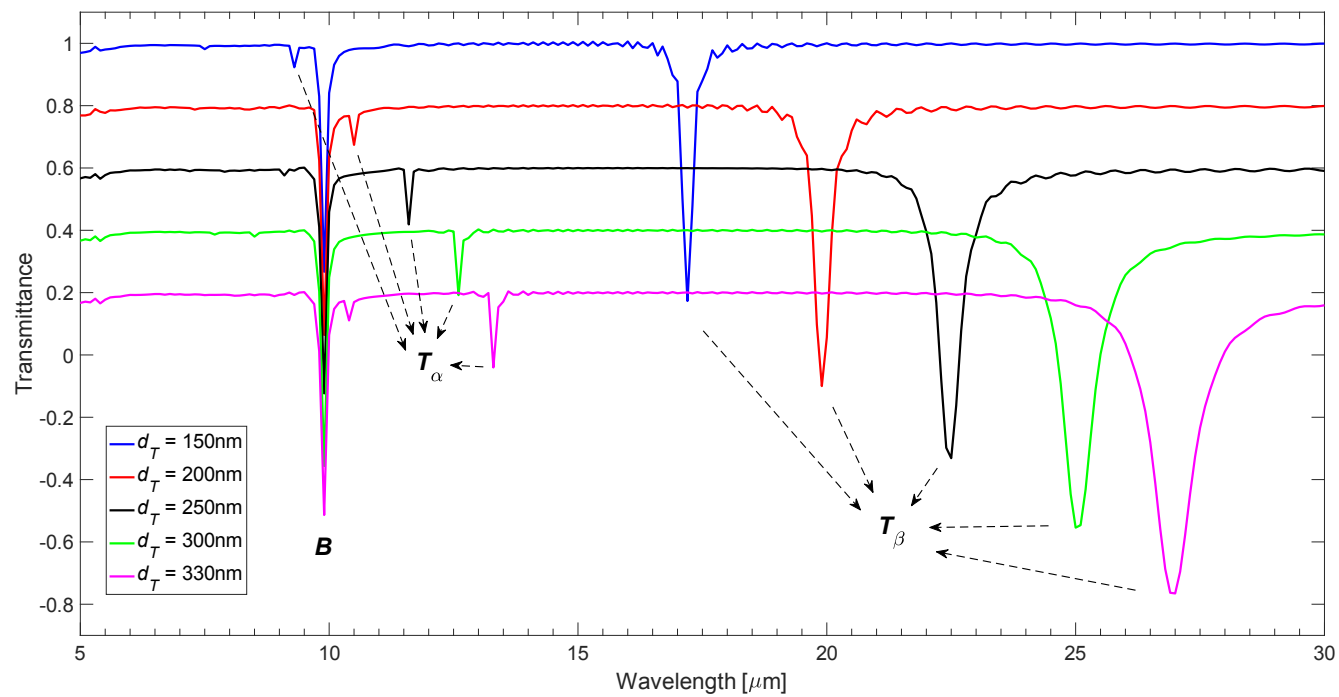

Figure 10: Wavelength-dependent light transmittance determined from the structure shown in Fig.1. In the simulation $\mu_{c}^{T}=0.20 \mathrm{eV}, \mu_{c}^{B}=0.40 \mathrm{eV}, N_{T}=N_{B}=1, d_{B}=$ $100 \mathrm{~nm}$, and $\Delta=600 \mathrm{~nm}$. For clarity, the curves are presented with an offset in the $y$-axis: $d_{T}=200 \mathrm{~nm}$ is offset by -0.2 , the $250 \mathrm{~nm}$ the -0.4 , the $300 \mathrm{~nm}$ the -0.6 , and the $330 \mathrm{~nm}$ the -0.8 .
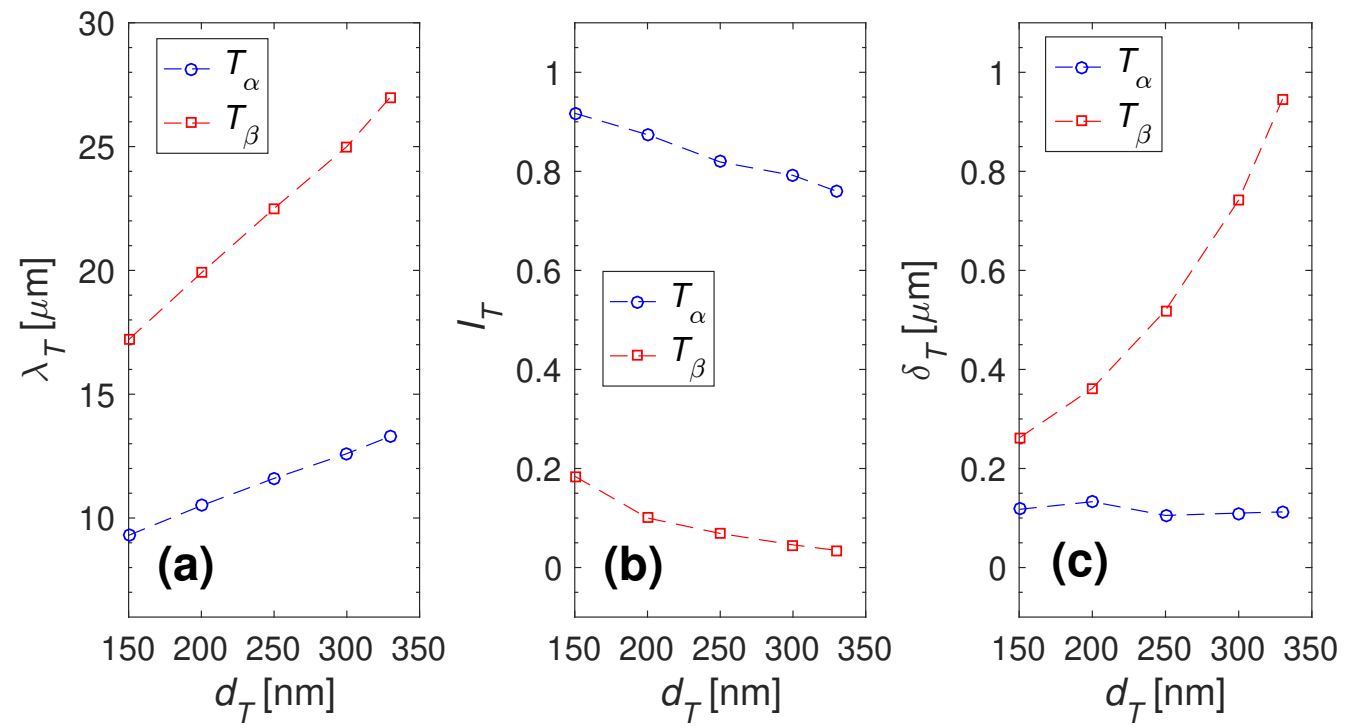

Figure 11: The values of the resonance wavelengths $\left(\lambda_{T}\right)$, the peak intensities $\left(I_{T}\right)$, and the FWHMs $\left(\delta_{T}\right)$, determined from peaks $T_{\alpha}$ and $T_{\beta}$ shown in Fig.10. 


\section{Declarations}

Ethics approval (The authors assure that this material is the authors' own original work, which has not been previously published elsewhere. The paper is not currently being considered for publication elsewhere. The paper reflects the authors' own research and analysis in a truthful and complete manner. The paper properly credits the meaningful contributions of co-authors and co-researchers. The results are appropriately placed in the context of existing research. All authors have been personally and actively involved in substantial work leading to the paper, and will take public responsibility for its content.)

Consent to participate (All authors agreed to participate in this research.)

Consent for publication (Permission from all the authors has been taken to publish this manuscript.)

Authors' contributions (All authors contributed to the design and implementation of the research, analysis of the results, and the manuscript's preparation.)

Funding (Not applicable)

Competing interests (The authors declare no competing interests.)

Availability of data and material (All data generated or used during the study appear in the submitted article.) 


\section{References}

\section{References}

[1] Pala RA, Shimizu KT, Melosh NA, Brongersma ML (2008) A nonvolatile plasmonic switch employing photochromic molecules. Nano Letters 8:15061510 .

[2] MacDonald KF, Samson ZL, Stockman MI, Zheludev NI (2009) Ultrafast active plasmonics. Nature Photonics 3:55-58.

[3] Dionne JA, Diest K, Sweatlock LA, Atwater HA (2009) PlasMOStor: A metal-oxide-Si field effect plasmonic modulator. Nano Letters 9:897-902.

[4] Liu M, Yin XB, Ulin-Avila E, Geng B, Zentgraf T, Ju L, Wang F, Zhang X (2011) A graphene-based broadband optical modulator. Nature 474:6467.

[5] Yan H, Li X, Chandra B, Tulevski G, Wu YQ, Freitag M, Zhu WJ, Avouris P, Xia FN (2012) Tunable infrared plasmonic devices using graphene/insulator stacks. Nature Nanotechnology 7:330-334.

[6] Liu PH, Cai W, Wang L, Zhang XZ, Xu JJ (2012) Tunable terahertz optical antennas based on graphene ring structures. Applied Physics Letters 100:153111.

[7] Dash S, Soni G, Patnaik A, Liaskos C, Pitsillides A, Akyildiz IF (2021) Switched-beam graphene plasmonic nanoantenna in the Terahertz wave region. Plasmonics https://doi.org/10.1007/s11468-021-01449-y.

[8] Sun C (2018) On the plasmonic properties of Ag@SiO2@Graphene coreshell nanostructures. Plasmonics 13:1671-1680.

[9] Azar MTH, Zavvari M, Zehforoosh Y, Mohammadi P (2020) Graphene plasmonic crystal: two-dimensional gate-controlled chemical potential for creation of photonic bandgap. Plasmonics 15:975-983.

[10] Monfared YE, Qasymeh M (2021) Graphene-assisted infrared plasmonic metamaterial absorber for gas detection. Results in Physics 23:103986.

[11] Grigorenko AN, Polini M, Novoselov KS (2012) Graphene plasmonics. Nature Photonics 6:749-758. 
[12] Bao Q, Loh KP (2012) Graphene photonics, plasmonics, and broadband optoelectronic devices. ACS Nano 6:3677-3694.

[13] Dash S, Patnaik A (2018) Material selection for $\mathrm{THz}$ antennas. Microw Opt Technol Lett 60(5):1183-1187.

[14] Jablan M, Buljan H, Soljacic M (2009) Plasmonics in graphene at infrared frequencies. Physical Review B 80:245435.

[15] Gao WL, Shu J, Qiu CY, Xu QF (2012) Excitation of plasmonic waves in graphene by guided-mode resonances. ACS Nano 6:7806-7813.

[16] Ju L, Geng BS, Horng J, Girit C, Martin M, Hao Z, Bechtel HA, Liang XG, Zettl A, Shen YR, Wang F (2011) Graphene plasmonics for tunable terahertz metamaterials. Nature Nanotechnology 6:630-634.

[17] Sun C, Wang XQ (2017) Plasmonic tuning at mid-infrared wavelengths by composite arrays of graphene ribbons. Plasmonics 12:1235-1243.

[18] Liu JQ, Wang DY, Wu S, He MD, Yu LS, Chao XB, Sun GH (2017) Tunable plasmonic dispersion and strong coupling in graphene ribbon and double layer sheets structure. Plasmonics 12:309-314.

[19] Wang PJ, Ding J, Chen WW, Dai SX, Zhang BH, Lu H, Fu Q, Li J, Li Y, Dai TG, Wang YH, Yu H, Yang JY (2020) Terahertz plasmonic SWAP and Fredkin gates utilizing graphene nano-ribbon waveguides. Optics Communications 463:125397.

[20] Hu L, Cheng FY, Tang YX, Wang HJ (2021) Giant and dynamically tunable plasmonic circular dichroism in graphene ribbons and Z-shaped metal metamaterials. The European Physical Journal B 94:8.

[21] FDTD Solutions, www.lumerical.com.

[22] Hanson GW (2008) Dyadic Green's functions and guided surface waves for a surface conductivity model of graphene. Journal of Applied Physics 103:064302.

[23] Stauber T, Peres NMR, Guinea F (2007) Electronic transport in graphene: A semiclassical approach including midgap states. Physical Review B 76:205423. 
[24] Tan YW, Zhang Y, Bolotin K, Zhao Y, Adam S, Hwang EH, Sarma SD, Stormer HL, Kim P (2007) Measurement of Scattering Rate and Minimum Conductivity in Graphene. Physical Review Letters 99:246803.

[25] Chu HS, Gan CH (2013) Active plasmonic switching at mid-infrared wavelengths with graphene ribbon arrays. Appllied Physics Letters 102:231107.

[26] Gusynin VP, Sharapov SG, Carbotte JP (2007) Sum rules for the optical and Hall conductivity in graphene. Physical Review B 75:165407.

[27] Casiraghi C, Hartschuh A, Lidorikis E, Qian H, Harutyunyan H, Gokus T, Novoselov KS, Ferrari AC (2007) Rayleigh Imaging of Graphene and Graphene Layers. Nano Letters 7:2711-2717.

[28] Kim M, Lee S, Kim S (2015) Plasmon-induced transparency in coupled graphene gratings. Plasmonics 10:1557-1564. 


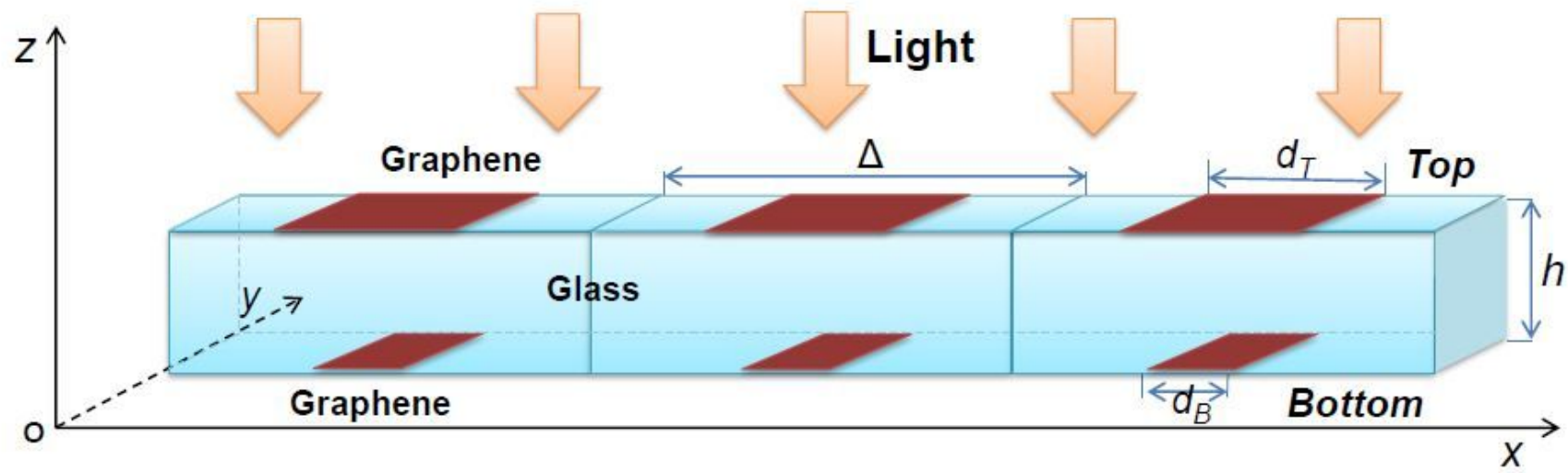

\section{Figure 1}

Schematic of the double-deck structure consisting of graphene nanoribbons. A plane-wave light is normally incident along the -z-axis. Two sets of graphene ribbon arrays (indicated in Top and Bottom) are placed on both sides of a glass substrate. The thickness of the glass is $h$. Note that along the x-axis, the basic structure in one period repeats innitely to full the array condition; only three periods are drawn in the gure for brevity. The length of one period along the x-axis is $\Delta$. The ribbons are innite in the y direction. The widths of the ribbons are $\mathrm{dT}$ and $\mathrm{dB}$, respectively.

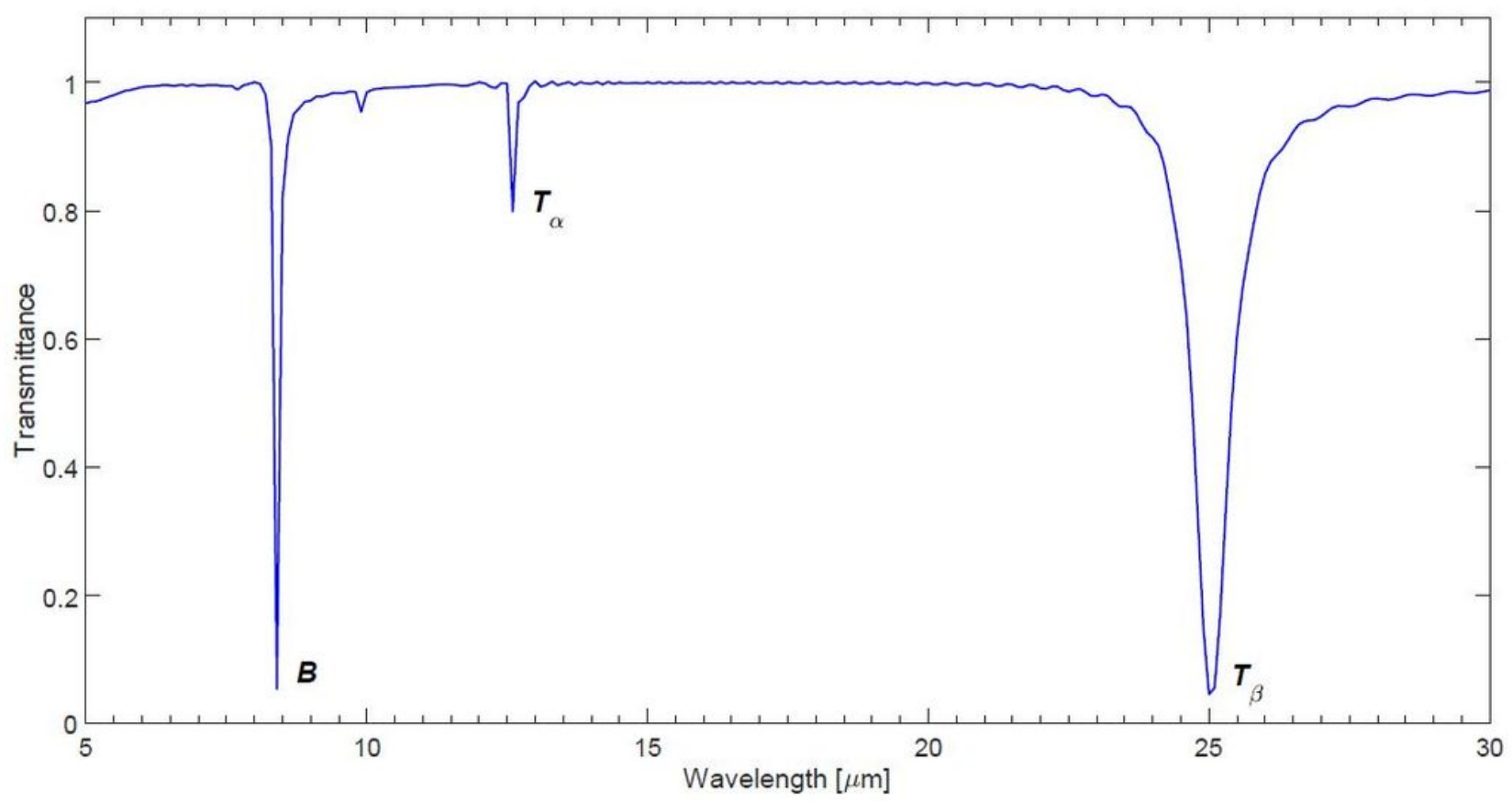

Figure 2 
Wavelength-dependent light transmittance determined from the structure shown in Fig.1. In the simulation $\mu \mathrm{Tc}=0.20 \mathrm{eV}, \mu \mathrm{Bc}=0.55 \mathrm{eV}, \mathrm{NT}=\mathrm{NB}=1, \mathrm{dT}=300 \mathrm{~nm}, \mathrm{~dB}=100 \mathrm{~nm}$, and $\Delta=600 \mathrm{~nm}$.
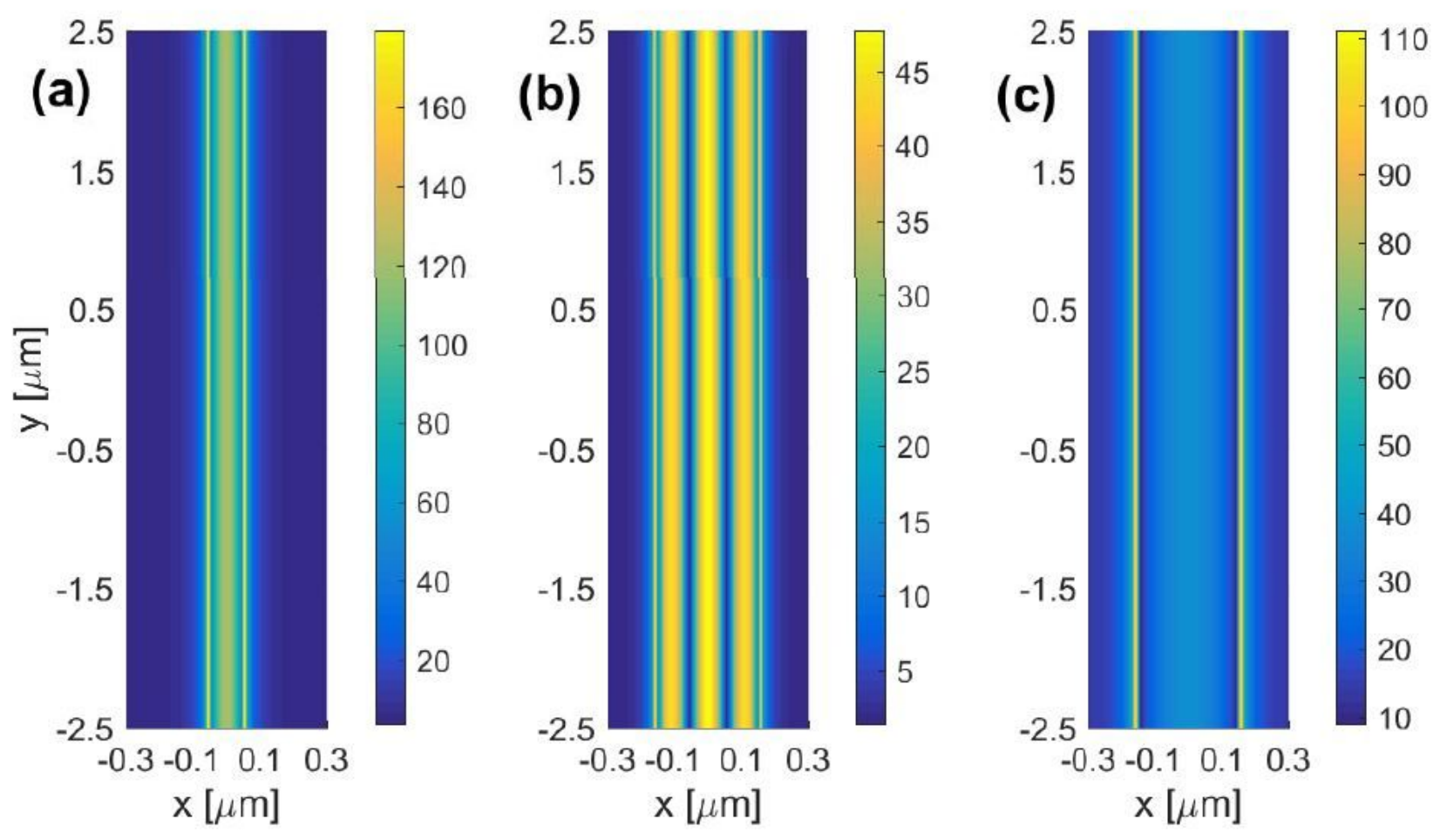

\section{Figure 3}

Distribution of the electric eld intensity at the surface of (a) Bottom graphene, and (b) and (c) Top graphene in the $x-y$ plane. The wavelength of the incident light was at (a) peak B, (b) peak Ta, and (c) peak $\mathrm{T}$ indicated in Fig.2. In the simulation $\mu \mathrm{Tc}=0.20 \mathrm{eV}, \mu \mathrm{Bc}=0.55 \mathrm{eV}, \mathrm{NT}=\mathrm{NB}=1, \mathrm{dT}=300 \mathrm{~nm}, \mathrm{~dB}=$ $100 \mathrm{~nm}$, and $\Delta=600 \mathrm{~nm}$. Along the $\mathrm{x}$-axis, the range from -0.3 to $0.3 \mu \mathrm{m}$ represents one period of the array, where the Bottom graphene ribbon was located within $(-0.050 .05) \mu \mathrm{m}$ and the Top ribbon within $(-0.15$ $0.15) \mu \mathrm{m}$. Note that the intensity of the color bar is different in each gure. 


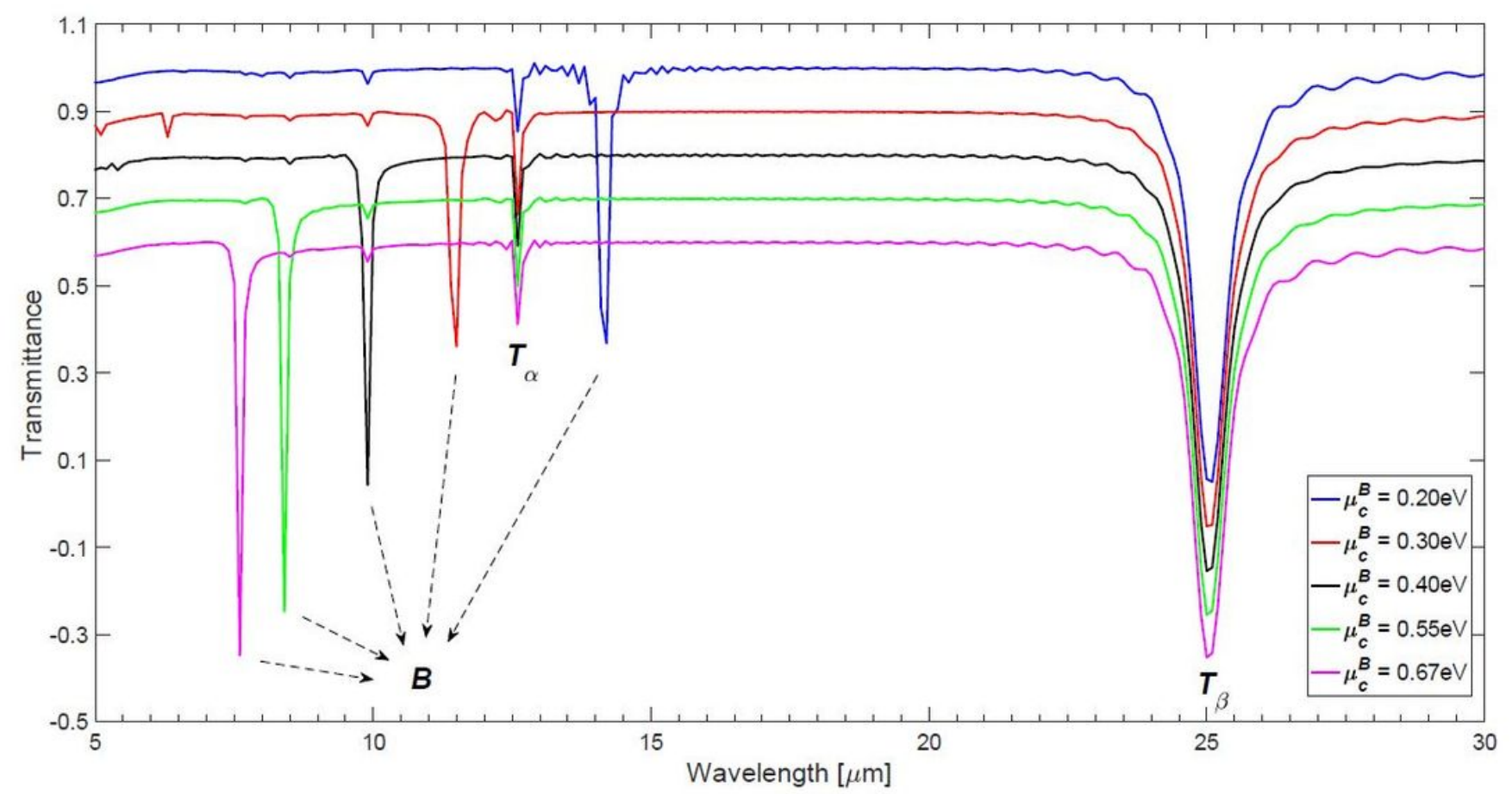

Figure 4

Wavelength-dependent light transmittance determined from the structure shown in Fig.1. In the simulation $\mu \mathrm{Tc}=0.20 \mathrm{eV}, \mathrm{NT}=\mathrm{NB}=1, \mathrm{dT}=300 \mathrm{~nm}, \mathrm{~dB}=100 \mathrm{~nm}$, and $\Delta=600 \mathrm{~nm}$. For clarity, the curves are presented with an offset in the $y$-axis: $\mu \mathrm{Bc}=0.30 \mathrm{eV}$ is offset by -0.1 , the $0.40 \mathrm{eV}$ the -0.2 , the $0.55 \mathrm{eV}$ the -0.3 , and the $0.67 \mathrm{eV}$ the -0.4 .
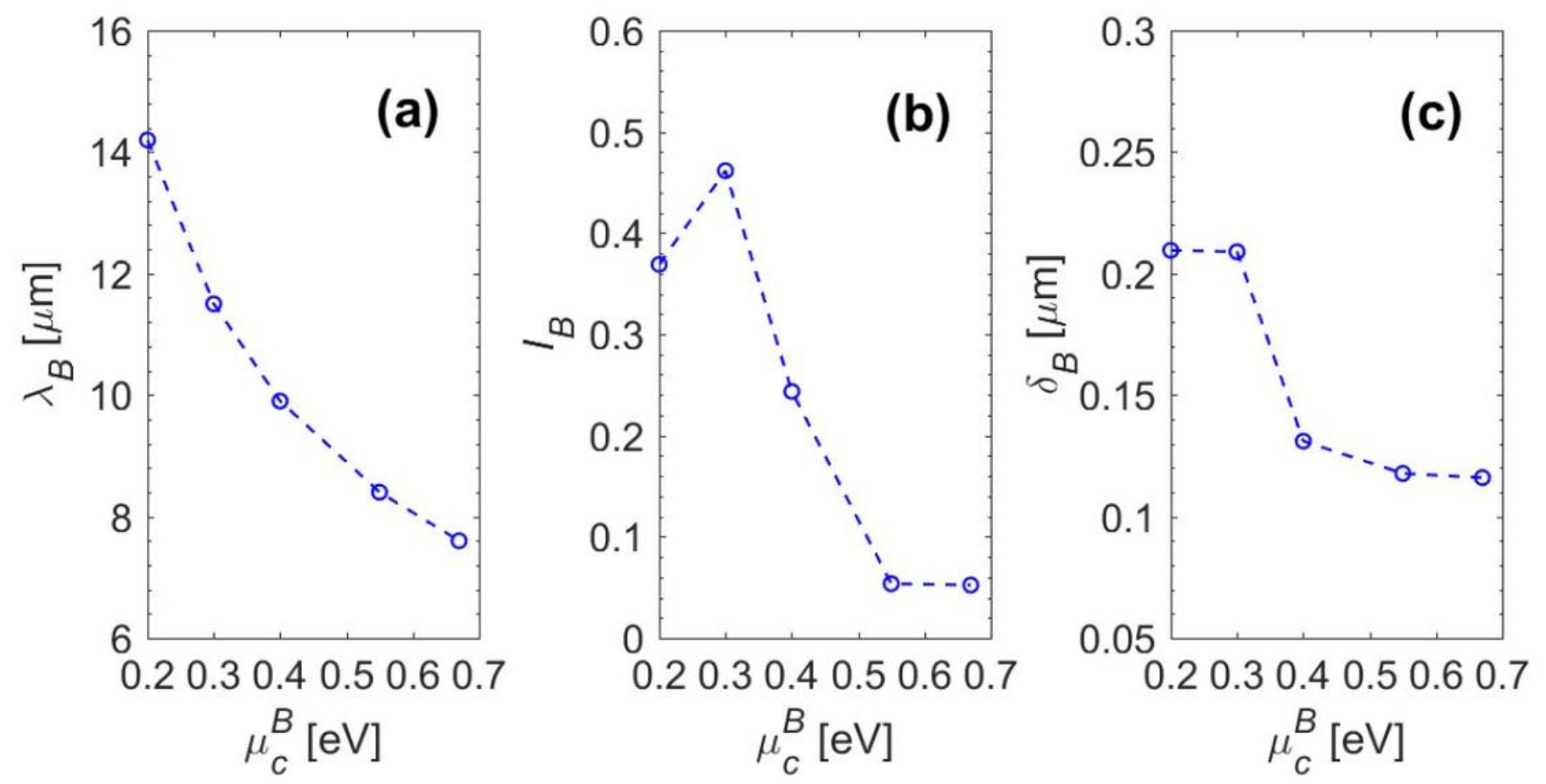

Figure 5 
The values of the resonance wavelength $(\lambda B)$, the peak intensity $(I B)$, and the FWHM $(\delta B)$, determined from peak B shown in Fig.4.

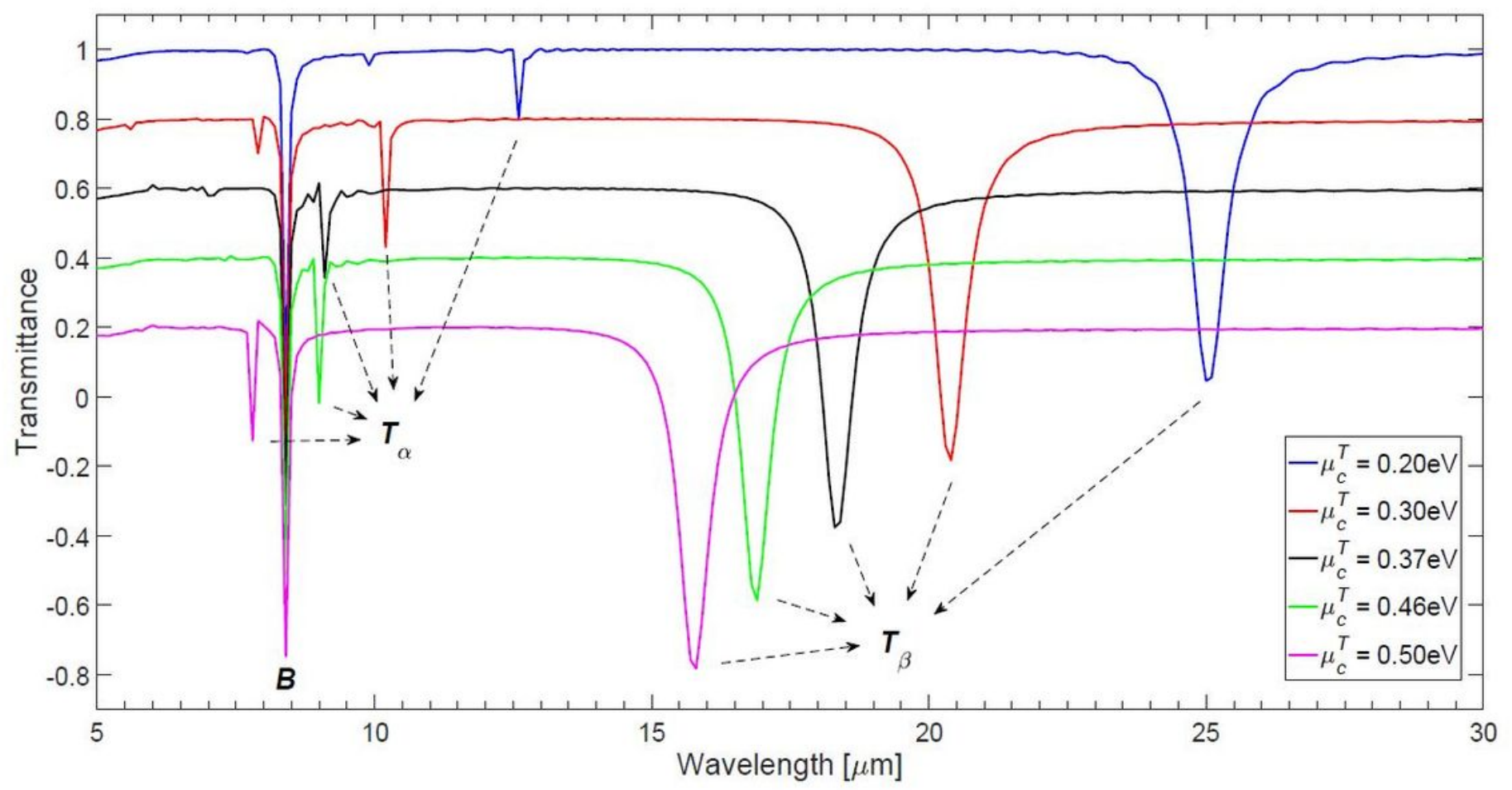

Figure 6

Wavelength-dependent light transmittance determined from the structure shown in Fig.1. In the simulation $\mu \mathrm{Bc}=0.55 \mathrm{eV}, \mathrm{NT}=\mathrm{NB}=1, \mathrm{dT}=300 \mathrm{~nm}, \mathrm{~dB}=100 \mathrm{~nm}$, and $\Delta=600 \mathrm{~nm}$. For clarity, the curves are presented with an offset in the $y$-axis: $\mu \mathrm{Tc}=0.30 \mathrm{eV}$ is offset by -0.2 , the $0.37 \mathrm{eV}$ the -0.4 , the $0.46 \mathrm{eV}$ the -0.6 , and the $0.50 \mathrm{eV}$ the -0.8 . 

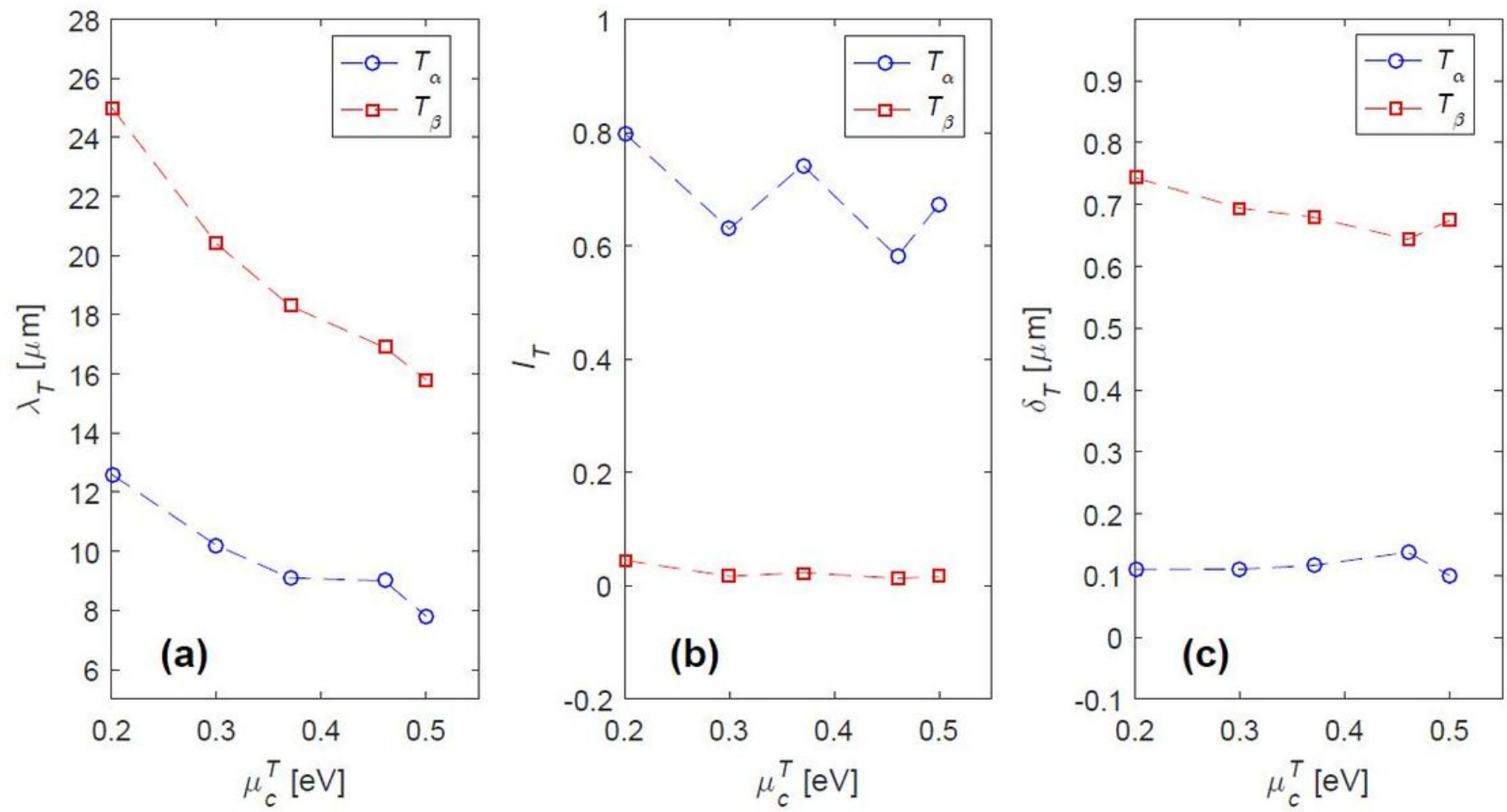

Figure 7

The values of the resonance wavelengths $(\lambda T$ ), the peak intensities (IT ), and the FWHMs ( $\delta T$ ), determined from peaks Ta and T shown in Fig.6.

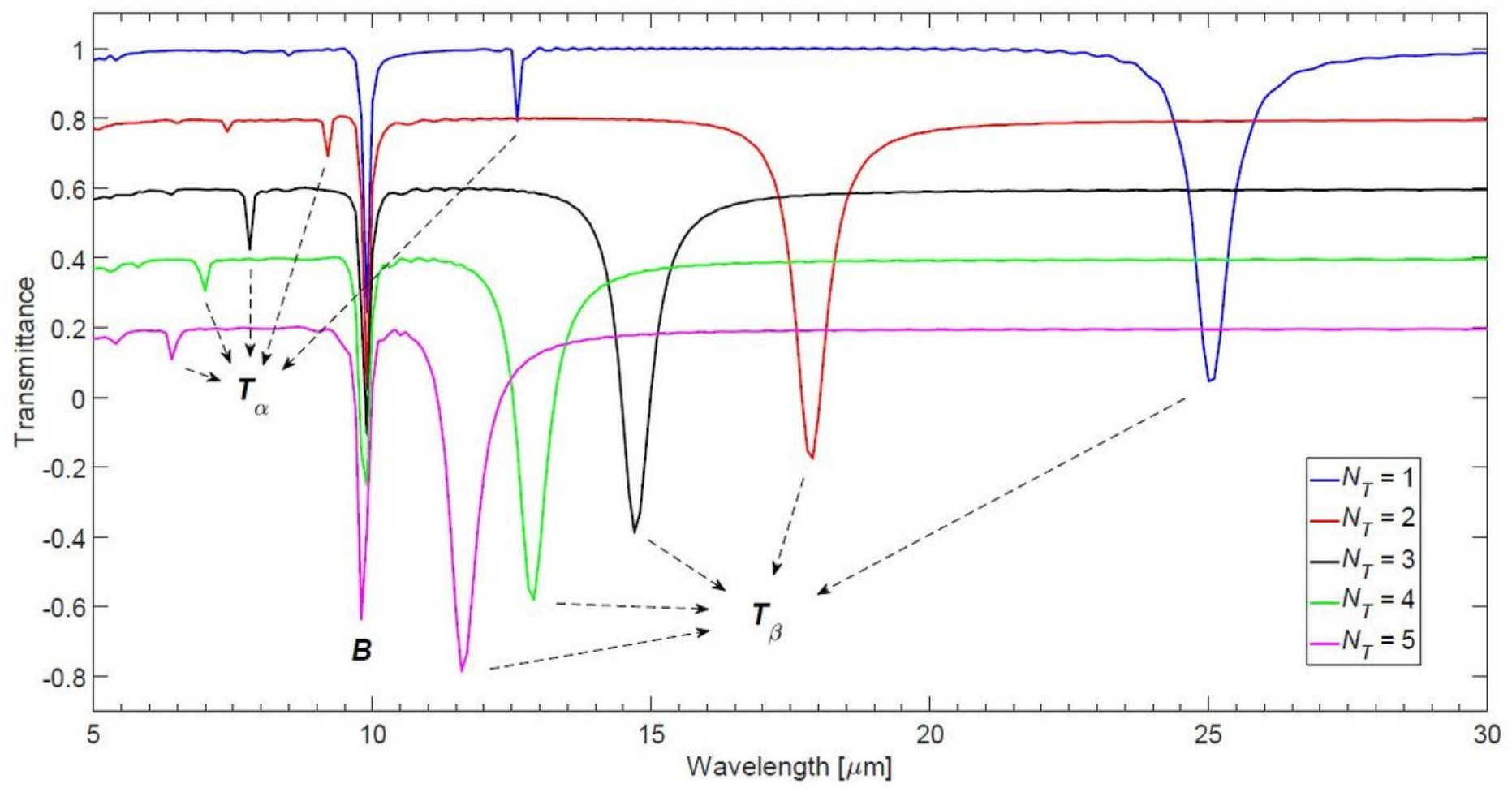

Figure 8 
Wavelength-dependent light transmittance determined from the structure shown in Fig.1. In the simulation $\mu \mathrm{Tc}=0.20 \mathrm{eV}, \mu \mathrm{Bc}=0.40 \mathrm{eV}, \mathrm{NB}=1, \mathrm{dT}=300 \mathrm{~nm}, \mathrm{~dB}=100 \mathrm{~nm}$, and $\Delta=600 \mathrm{~nm}$. For clarity, the curves are presented with an offset in the y-axis: NT $=2$ is offset by -0.2 , the 3 the -0.4 , the 4 the -0.6 , and the 5 the -0.8 .
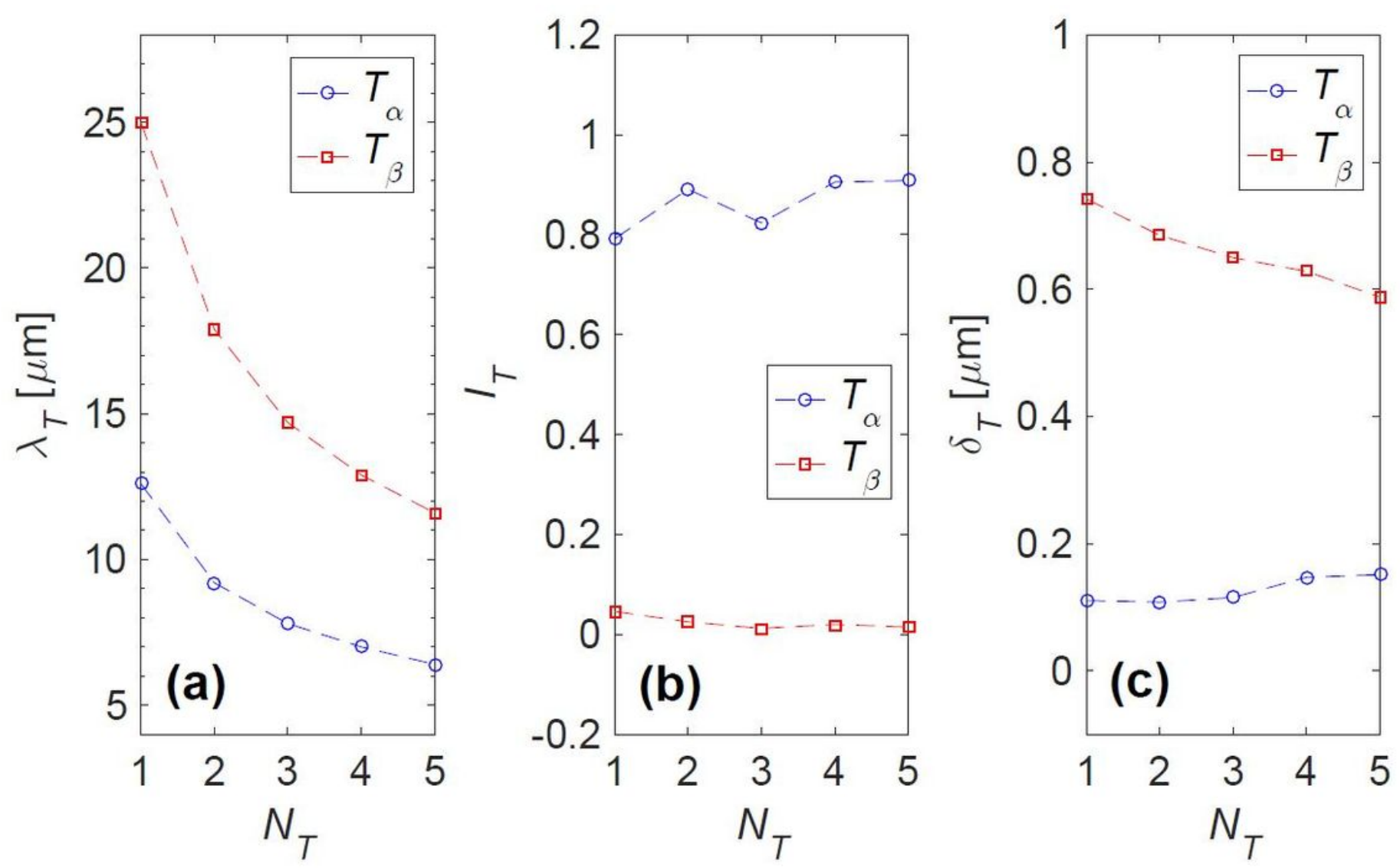

Figure 9

The values of the resonance wavelengths $(\lambda T$ ), the peak intensities (IT ), and the FWHMs ( $\delta T$ ), determined from peaks Ta and T shown in Fig.8. 


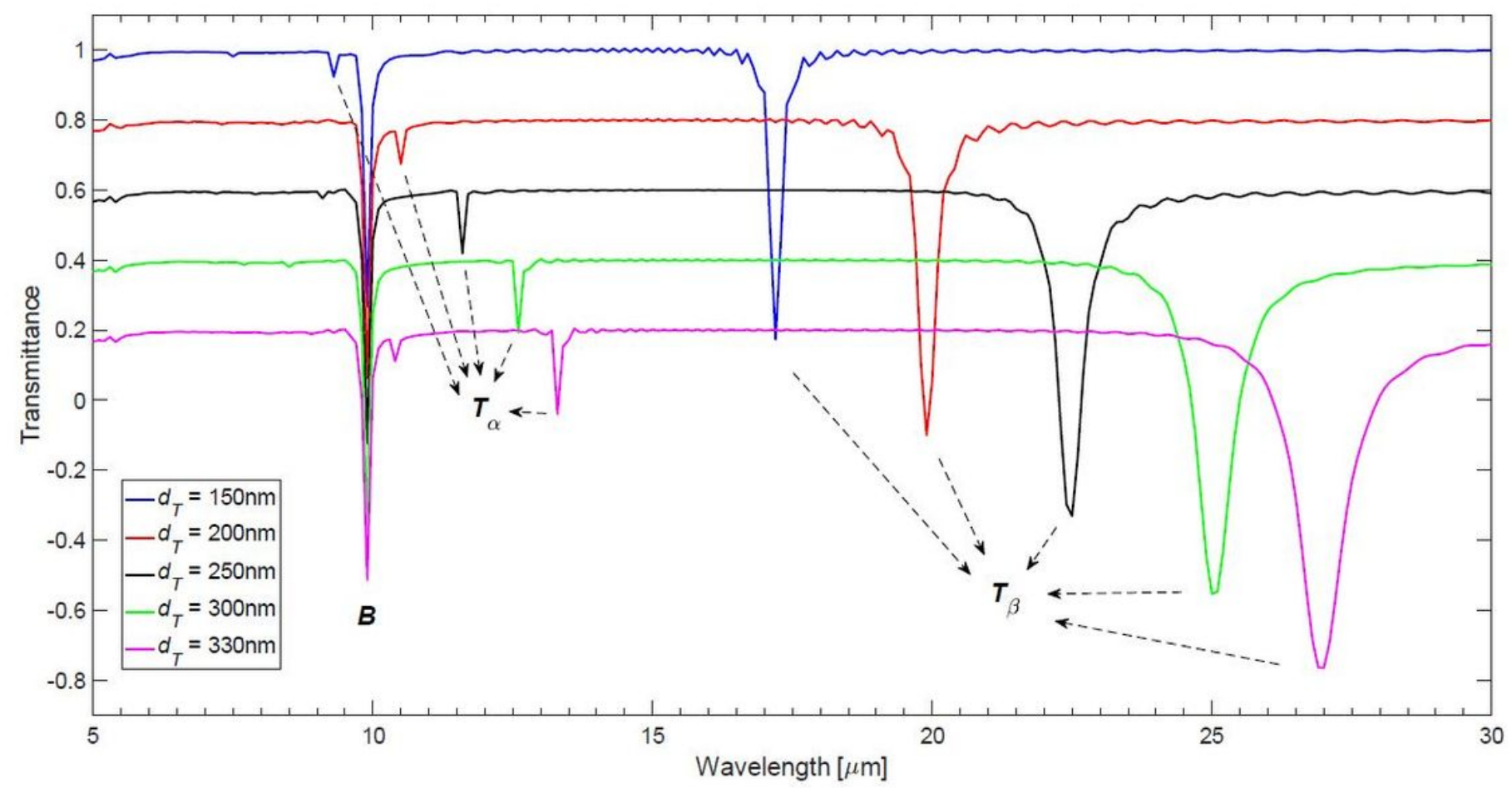

Figure 10

Wavelength-dependent light transmittance determined from the structure shown in Fig.1. In the simulation $\mu \mathrm{Tc}=0.20 \mathrm{eV}, \mu \mathrm{Bc}=0.40 \mathrm{eV}, \mathrm{NT}=\mathrm{NB}=1, \mathrm{~dB}=100 \mathrm{~nm}$, and $\Delta=600 \mathrm{~nm}$. For clarity, the curves are presented with an offset in the y-axis: $d T=200 \mathrm{~nm}$ is offset by -0.2 , the $250 \mathrm{~nm}$ the -0.4 , the $300 \mathrm{~nm}$ the -0.6 , and the $330 \mathrm{~nm}$ the -0.8 . 

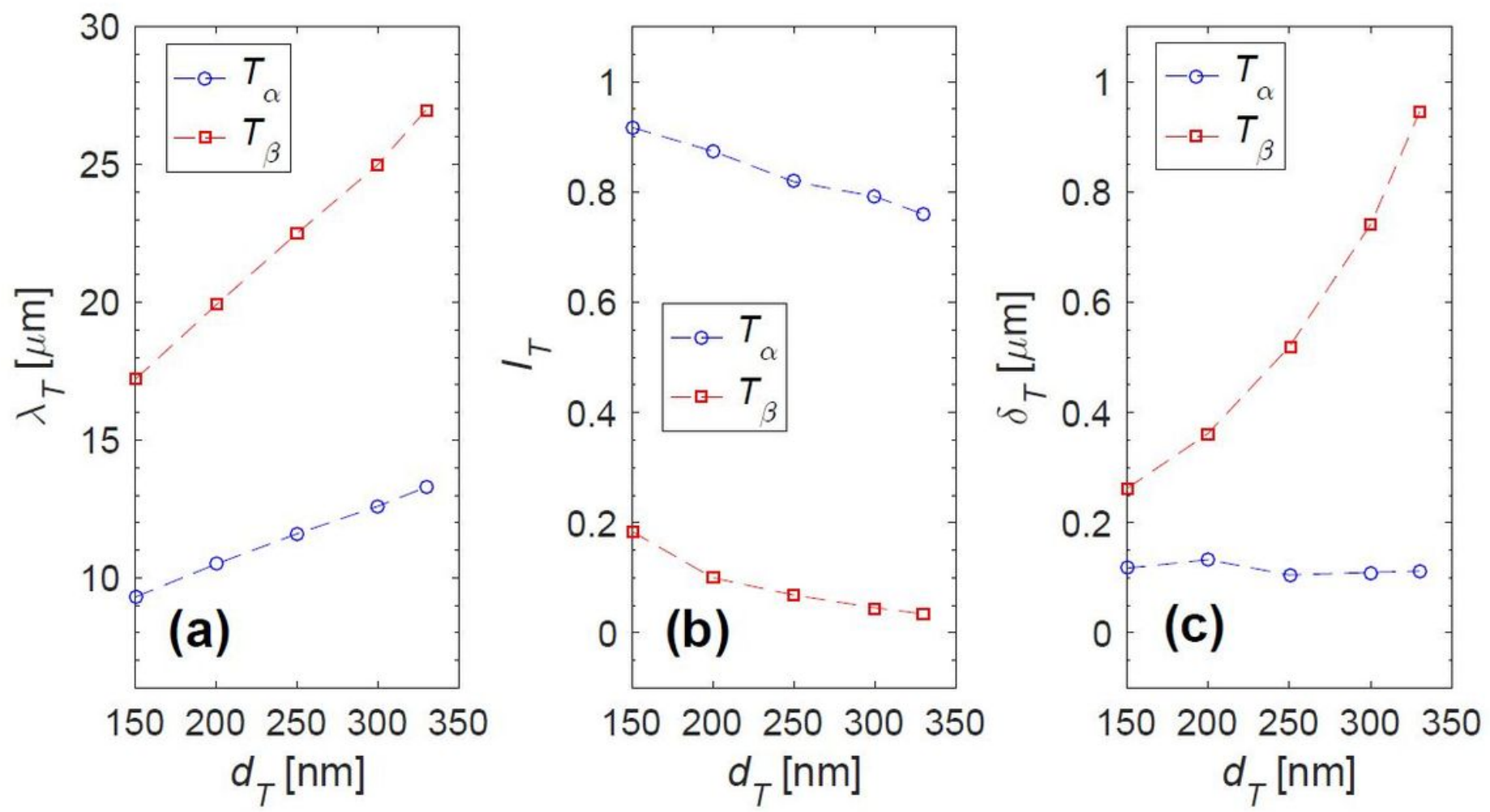

Figure 11

The values of the resonance wavelengths $(\lambda T$ ), the peak intensities (IT ), and the FWHMs ( $\delta T$ ), determined from peaks Ta and T shown in Fig.10. 